\title{
Intravascular extranodal NK/T-cell lymphoma, nasal type, presenting as acute abdomen
}

\author{
Serge Alexanian • Anna K. Wong • Jonathan Said • \\ Chisa Aoyama • Albert Park • Sheeja T. Pullarkat
}

Received: 16 September 2010 / Accepted: 8 November 2010 / Published online: 23 November 2010

(C) Springer-Verlag 2010

\begin{abstract}
Intravascular lymphomas (IVLs) overwhelmingly express B-cell phenotypes, although $\mathrm{T}$ lineage is not uncommon. However, purely intravascular NK/T-cell lymphomas are vanishingly rare. We present a case of intravascular extranodal NK/T-cell lymphoma (ENKTCL), nasal type, in a 37-year-old Hispanic man admitted for severe abdominal pain. Intraoperative evaluation revealed necrotic small bowel and multiple mesenteric venous thromboses. Initial histologic examination demonstrated classic changes of ischemic bowel without evidence of dense lymphoid infiltrates or other mass lesions. However closer review of the mesenteric vasculature exposed an angioinvasive and angiodestructive process comprised of lymphomatous cells which were positive by immunohistochemistry for CD2, CD3, CD8, BCL2, TIA-1, Granzyme $\mathrm{B}$, and Epstein-Barr virus (EBV) EBER in situ hybridization. Although exceedingly rare, isolated intravascular
\end{abstract}

S. Alexanian $\cdot$ J. Said $\cdot$ S. T. Pullarkat $(\bowtie)$

Department of Pathology and Laboratory Medicine, David Geffen

School of Medicine at University of California-Los Angeles,

10833 Le Conte Avenue, A7-149 CHS, Mail Code: 173216,

Los Angeles, CA 90095-1732, USA

e-mail: SPullarkat@mednet.ucla.edu

\section{A. K. Wong}

Department of Pathology and Laboratory Medicine, University of California-San Diego,

San DiegoCA, USA

C. Aoyama

Department of Pathology and Laboratory Medicine, Los Angeles County-Olive View-Medical Center, Los Angeles, CA, USA

\section{A. Park}

Department of Hematology and Oncology, Los Angeles County-Olive View-UCLA Medical Center, Los Angeles, CA, USA
ENKTCL, nasal type, may present without a mass; such lesions must be recognized since IVLs typically exhibit widespread dissemination and portend a dire prognosis with most patients inevitably succumbing to their disease.

Keywords NK/T cell lymphoma $\cdot$ Intravascular lymphoma $\cdot$ Acute abdomen $\cdot$ EBV-EBER

\section{Background}

Extranodal NK/T-cell lymphoma (ENKTCL), nasal type, is a rare neoplasm with a predisposition for the nasal cavity and paranasal sinuses and hence was formally known as lethal midline granuloma or polymorphic reticulosis $[1,2]$. While relatively uncommon in North America and Europe, it demonstrates a marked predilection for Asians and Central/South Americans [1,2]. The characteristic histologic feature is an angiocentric/ angiodestructive growth pattern with zonal necrosis [1,2]. Extranasal ENKTCL shows the same histologic characteristics but occurs as a locally destructive space occupying mass lesion in other sites such as the skin, or, less commonly, the gastrointestinal tract, spleen, and testes [3-5]. These cases are typically associated with a higher proportion of disseminated disease, aggressive clinical courses, unfavorable outcomes and strong association with Epstein-Barr virus (EBV) $[6,7]$. Although the cell of origin is often the natural killer (NK) cell, some cases arise from cytotoxic $\mathrm{T}$ cells (NK-like T cells that express CD56 and TIA-1), and hence the term NK/T-cell lymphoma was adopted $[1,2]$.

Intravascular lymphoma (IVL) is a rare disorder characterized by the presence of large neoplastic lymphoid cells restricted to the lumens of small vessels, particularly capillaries. The World Health Organization (WHO) classi- 


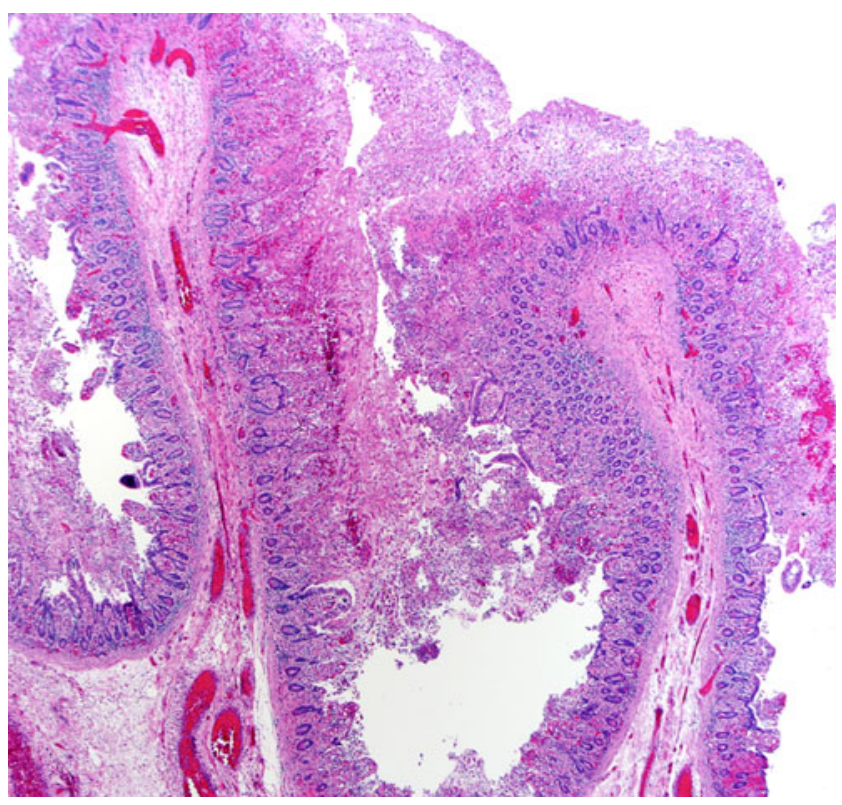

Fig. 1 The small bowel mucosa shows transmural ischemic changes. The mucosal surface is covered with an exudate composed of fibrin and neutrophils $(\mathrm{H} \& \mathrm{E}$, original magnification $\times 40)$

fication has subcategorized IVL as a variant of extranodal diffuse large B-cell lymphoma as the overwhelming majority of reported cases demonstrate a B-cell phenotype [8]. Clinically, IVL may present with localized symptoms, particularly cutaneous or neurological, although disseminated multiorgan failure is more common in Asian populations [8].

T- or NK-cell derived lymphomas with a strictly intravascular pattern of involvement are exceptionally rare. Here, we present one such case of a purely intravascular ENKTCL presenting in a unique fashion: a surgical acute abdomen.

\section{Report of a case}

A 37-year-old Hispanic man with past medical history significant for hypertension presented to the emergency department with severe abdominal pain and mild leukocytosis. Computed tomography imaging of the abdomen showed massive ascites and thickened small bowel loops. No mass lesions were identified. In addition, the patient also complained of vision loss and imaging studies showed bilateral retinal detachment without any space occupying lesion. The patient underwent emergent exploratory laparotomy and intraoperative examination revealed a large segment of necrotic small bowel and multiple mesenteric small bowel venous thromboses as detected by intraoperative ultrasound. Mesenteric arteries were patent and unremarkable. Approximately $300 \mathrm{~cm}$ of small bowel was resected, followed by primary anastomosis.
A diagnosis of an ENKTCL, nasal type, was rendered upon microscopic examination of the resected bowel aided by immunohistochemistry. Additional workup including an MRI of the brain and orbit failed to reveal the cause for the bilateral retinal detachment. The patient was started on a modified SMILE regimen comprised of prednisone, Ifosafamide, L-asparaginase, and etoposide without methotrexate.

\section{Materials and methods}

Immunohistochemical analysis of the stains was performed on formalin-fixed and paraffin embedded $4-\mu \mathrm{m}$ sections. The tissue sections were deparaffinized and treated with $0.5 \%$ hydrogen peroxide to block endogenous peroxidase activity and then heated in a vegetable steamer in $10 \mathrm{mM}(0.01 \mathrm{M})$ citric acid $(\mathrm{pH}$ 6.0) for $25 \mathrm{~min}$ at $97^{\circ} \mathrm{C}$ followed by slow cooling for antigen retrieval. Slides were then incubated for 30 minutes at room temperature with monoclonal antibodies against CD2 (Novocastro/ Leica Microsystems, Bannockburn, IL), CD3 (PS1, Biocare Medical, Concord, CA), CD4 (Neomarkers/Labvision, Freemont, CA), CD5 (Ventana Medical Systems, Tucson, AZ), CD7 (Biocare Medical), CD8 (DAKO Corp, Carpinteria, CA), CD20 (DAKO Corp), CD56 (Biocare Medical), TIA-1 (Beckman Coulter, Brea, CA), Granzyme B (DAKO Corp), and EBVEBER (Ventana Medical Systems ). The slides were subsequently stained with an Ultravision LP detection

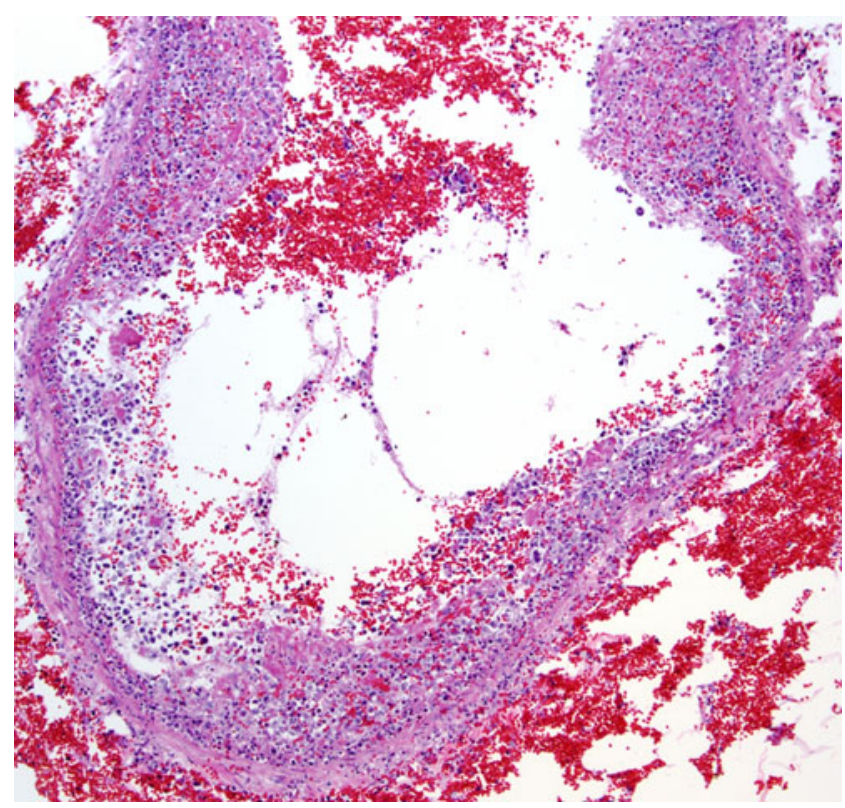

Fig. 2 Some submucosal mesenteric vessels contained large lymphoma cells without complete luminal occlusion (H\&E, original magnification $\times 100$ ) 


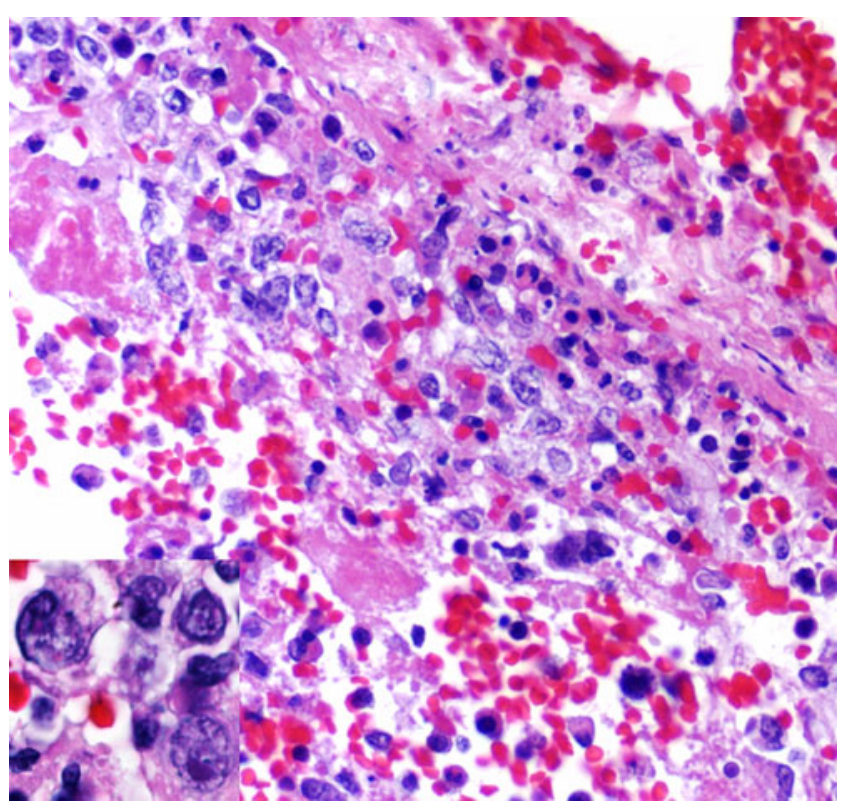

Fig. 3 Mesenteric vessels contained large lymphoma cells marginating along the luminal wall with admixed neutrophils. Mitotic and apoptotic figures are readily identified (H\&E, original magnification $\times$ 400). Inset: high $\mathrm{N} / \mathrm{C}$ ratios, scanty cytoplasm, irregular nuclear contours $(\mathrm{H} \& \mathrm{E}, \times 1000)$

system (Thermo Scientific, Freemont, CA) using diaminobenzidine as the chromogen and hematoxylin as the counterstain.

\section{Results}

\section{Macroscopic}

The partial enterectomy specimen was received in formalin and measured $300 \mathrm{~cm}$ in length. The bowel wall ranged in thickness from 0.5 to $0.7 \mathrm{~cm}$. Grossly the mucosal surface appeared hemorrhagic with luminal blood clots and edematous folds. No perforations or mass lesions were identified.

Table 1 Summary of the immunohistochemical profile of the intravascular lesion

\begin{tabular}{ll}
\hline Positive & Negative \\
\hline CD2 & CD4 CD20 \\
CD3 & CD5 \\
CD8 & CD7 \\
TIA-1 & CD20 \\
Granzyme B & PAX-5 \\
EBV by in situ hybridization & CD34 \\
& CD56 \\
& CD117 \\
& ALK \\
\hline
\end{tabular}

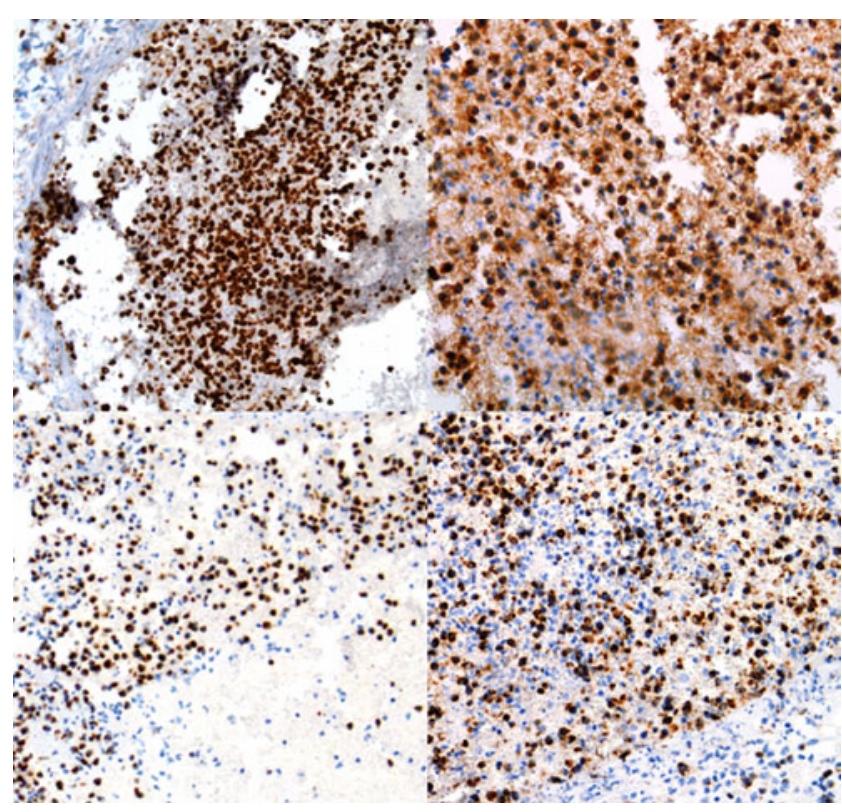

Fig. 4 Clockwise starting from top left: lymphoma cells are CD3 positive, CD2 positive, Granzyme-B positive, and TIA-1 positive (original magnification $\times 100$ )

Histologic and immunophenotypic findings

Histologic sections demonstrated transmural ischemic changes, hemorrhage, serositis, nonspecific inflammatory infiltrate of the lamina propria and congestion and dilatation of submucosal vessels with necrosis (Fig. 1). Closer inspection revealed that medium-sized mesenteric vessels were infiltrated by angioinvasive and angiodestructive

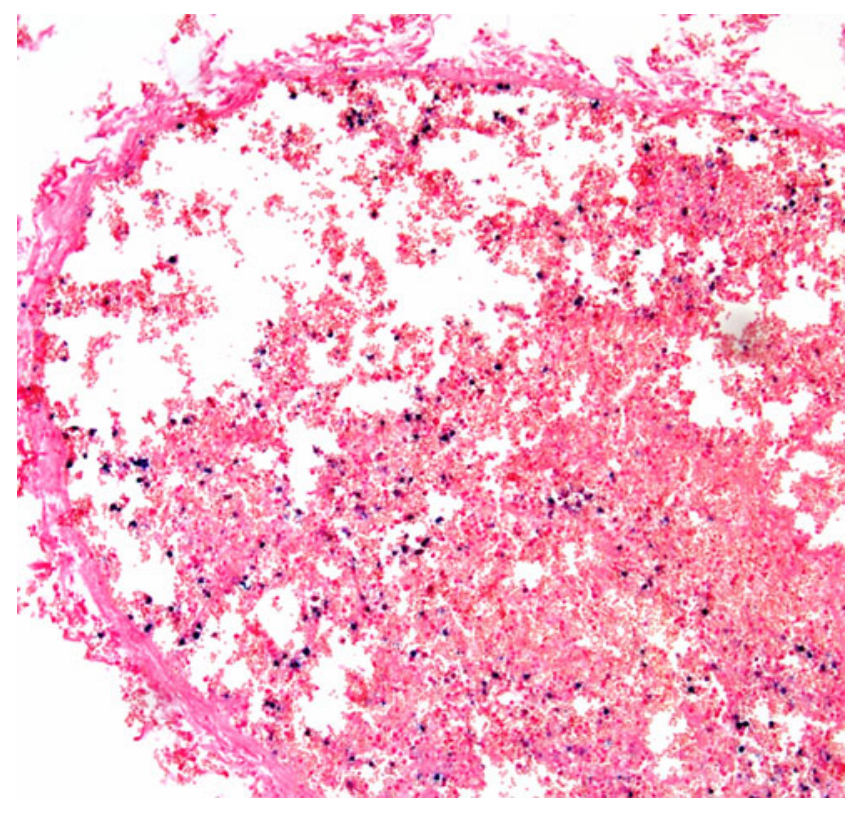

Fig. 5 Epstein-Barr virus (EBV) in situ hybridization (EBV-encoded RNA, blue) demonstrates scattered EBV positive lymphoma cells (original magnification $\times 100$ ) 
atypical lymphocytic cells associated with fibrinoid necrosis and a background of numerous neutrophils and apoptotic figures (Fig. 2). These atypical cells were medium to large and exhibited high nuclear to cytoplasmic ratios, scant clear cytoplasm, irregular nuclear contours, two to three small nucleoli, and vesicular chromatin (Fig. 3). Of note, the atypical cells were entirely restricted to vascular lumens and the remainder of the colonic wall failed to show any atypical lymphoid infiltrate.

On immunohistochemistry (Table 1), the atypical intravascular lymphoid infiltrate was positive for CD3, CD2, TIA-1, and Granzyme B (Fig. 4) but negative for CD4, CD5, CD7, CD56, and CD20. In situ hybridization studies for EBV (EBV-encoded RNA) demonstrated strong nuclear positivity (Fig. 5). T-cell receptor genes exhibited a germline configuration with no clonal rearrangement.

\section{Discussion}

Primary lymphomas of the gastrointestinal tract account for 4-20\% of all non-Hodgkin lymphomas and are almost always of B-cell lineage. Rare NK/T-cell lymphomas involving the gastrointestinal tract have been described but constitute less than $1 \%$ of total reported cases; nearly all were associated with a discrete mass lesion $[4,5]$. Review of the published literature to date reveals this to be the first reported case of ischemic bowel disease secondary to a purely intravascular NK/T-cell lymphoma of the gastrointestinal tract. Furthermore, our patient presented with bilateral loss of vision. While both ocular and brain imaging remained negative for a discrete mass in our case, the bilateral retinal detachment remains quite suspicious for lymphoma given the focality and vasculocentric nature of this neoplasm.

IVL is a rare neoplasm almost uniformly demonstrating a B-cell immunophenotype and thus is regarded as a variant of extranodal diffuse large B-cell lymphoma by the WHO [8]. To our knowledge, NK/T-cell intravascular lymphoma (NK/TIVL) is vanishingly rare [9-15]. A review of these cases showed median age at presentation was 46 years with a male to female ratio of 1:2. Skin involvement was a predominant finding $(83 \%)$ while bone marrow involvement was seen in only one case [15]. The unifying feature in all these cases was EBV positivity. Outcomes were quite variable: one patient with localized disease who was treated with cyclophosphamide, doxorubicin, vincristine, and prednisone (CHOP) chemotherapy followed by stem cell transplantation achieved complete remission at the end of 1 year; three died from CNS involvement, multiorgan involvement, or acute graft versus host disease; two remained alive after the diagnosis, although follow-up was limited to 4 or 7 months [15].

Compared with NK/T-IVL, cases of intravascular B- and T-cell lymphomas typically present later, with a median age of 70 and 62 years, respectively. The female preponderance observed in NK-IVL is not present in either B- or T-cell IVL. Cutaneous involvement is present in $39 \%$ and $46 \%$ of cases of B- and T-cell IVL, respectively, compared with $83 \%$ of cases in NK/T-IVL. EBV positivity was rarely detected in B- and T-cell IVL [15].

In contrast to the previously reported cases of NK/TIVL, our patient had no cutaneous involvement. In addition, the microscopic pattern of vascular involvement was unique since the vessel walls showed marked destruction with increased neutrophils and apoptotic debris, a hallmark of ENKTCL, nasal type. In addition the lymphoma cells lined the intimal layer of the vessel wall without completely occluding the lumen, as is often the case in IVL (Figs. 2 and 3 ).

The clues to this case lay in the recognition of the curious history of mesenteric thromboses in an otherwise healthy patient, the cytomorphologic features of the atypical lymphoid cells, and their architectural predilection towards angioinvasion and angiodestruction. Features such as these must elicit increased diagnostic suspicion and lead to a more comprehensive immunohistochemical panel including NK-cell and cytotoxic markers as well as in situ hybridization studies for EBV EBER. Furthermore, although the classic atypical lymphoid infiltrate is angiocentric and leads to a certain degree of luminal narrowing by necrotic debris, it does not cause total luminal obstruction, as frequently seen with B-cell IVL $[9,15]$. Unfortunately, as this specific lymphoma exhibits a predilection for angioinvasion and angiodestruction, the tissue which is available for histologic review often demonstrates massive surrounding tissue necrosis and microbial superinfection, further complicating diagnosis [10].

The type of presentation we report reiterates the importance of maintaining diagnostic vigilance: an initial cursory review of the mesenteric vasculature with a limited immunohistochemical panel could have elicited a false diagnosis as CD3+ CD20- lymphoid infiltrates may have, in this particular case, been misinterpreted as a reactive/ vasculitic infiltrate. Recognition of this purely angioinvasive NK/T-cell lymphoma is clinically vital since patients presenting with disseminated disease in the absence of a discrete mass lesion display a more clinically aggressive course, and must be managed as such.

Conflicts of interest The authors declare that they have no conflict of interest.

\section{References}

1. Hasserjian RP, Harris NL (2007) NK-Cell lymphomas and leukemias: a spectrum of tumors with variable manifestations and immunophenotype. Am J Clin Pathol 127:860-868 
2. Chan JKC, Quintanilla-Martinez L, Ferry JA, Peh SC (2008) Extranodal NK/T-cell lymphoma, Nasal-type. In: Swerdlow SH et al (eds) WHO Classification of tumours of haematopoietic and lymphoid tissues, 4th edn. IARC, Lyon, pp 285-288

3. Ko YH, Cho EY, Kim JE, Lee SS, Huh JR, Chang HK, Yang WI, Kim CW, Kim SW, Ree HJ (2004) NK and NK-like T-cell lymphoma in extranasal sites: a comparative clinicopathological study according to site and EBV status. Histopathology 44(5):480-489

4. Kim JH, Lee JH, Lee J, Oh SO, Chang DK, Rhee PL, Kim JJ, Rhee JC, Lee J, Kim WS, Ko YH (2007) Primary NK-/T-cell lymphoma of the gastrointestinal tract: clinical characteristics and endoscopic findings. Endoscopy 39(2):156-160

5. Wakabayashi S, Arai A, Oshikawa G, Araki A, Watanabe M, Uchida N, Taniguchi S, Miura O (2009) Extranodal NK/T cell lymphoma, nasal type, of the small intestine diagnosed by doubleballoon endoscopy. Int J Hematol 90:605-610

6. Yim CY, Lee WS, Yuh YJ, Kim CW, Heo DS (2008) Clinical heterogeneity of extranodal NK/T-cell lymphoma, nasal type: a national survey of the Korean Cancer Study Group. Ann Oncol 19:1477-1484

7. Au WY, Weisenburger DD, Intragumtornchai T, Nakamura S, Kim WS, Sng I, Vose J, Armitage JO, Liang R (2009) International Peripheral T-Cell Lymphoma Project. Clinical differences between nasal and extranasal natural killer/T-cell lymphoma: a study of 136 cases from the International Peripheral T-Cell Lymphoma Project

8. Nakamura S, Ponzoni M, Campo E (2008) Intravascular large Bcell lymphoma. In: Swerdlow SH et al (eds) WHO classification of tumours of haematopoietic and lymphoid tissues, 4th edn. IARC, Lyon, pp 252-253

9. Cerroni L, Massone C, Kutzner H, Mentzel T, Umbert P, Kerl H (2008) Intravascular large T-cell or NK-cell lymphoma: a rare variant of intravascular large cell lymphoma with frequent cytotoxic phenotype and association with Epstein-Barr Virus infection. Am J Surg Pathol 32:891-898

10. Wu X, Li P, Zhao J, Yang X, Wang F, Yang YQ, Fang F, Xu Y, Zhang H, Wang WY, Yi C (2008) A clinical study of 115 patients with extranodal natural killer/t-cell lymphoma, nasal type. Clin Oncol 20:619-625

11. Wu H, Said J, Ames ED, Chen C, McWhorter V, Chen P, Ghali V, Pinkus GS (2005) First reported cases of intravascular large cell lymphoma of the NK cell type: clinical, histologic, immunophenotypic, and molecular features. Am J Clin Pathol 123:603-611

12. Santucci M, Pimpinelli N, Massi D et al (2003) Cytotoxic/natural killer cell cutaneous lymphomas. Report of EORTC cutaneous lymphomas task force workshop. Cancer 97:610-627

13. Kuo TT, Chen MJ, Kuo MC (2006) Cutaneous intravascular NKcell lymphoma: report of a rare variant associated with Epstein Barr virus. Am J Surg Pathol 30:1197-1201

14. Song DE, Lee MW, Ryu MH, Kang DW, Kim SJ, Huh J (2007) Intravascular large cell lymphoma of the natural killer cell type. J Clin Oncol 25:1279-1282

15. Nakamichi N, Fukuhara S, Aozasa K, Morii E (2008) NK-cell intravascular lymphomatosis - a mini-review. Eur J Haematol 81 (1):1-7 